\title{
Efeito do Tratamento das Fibras nas Propriedades do Biocompósito de Amido Termoplástico/Policaprolactona/Sisal
}

\author{
Adriana de Campos, Kelcilene B. R. Teodoro, José M. Marconcini, Luiz H. C. Mattoso \\ Laboratório Nacional de Nanotecnologia para o Agronegócio, Embrapa - São Carlos \\ Sandra M. Martins-Franchetti \\ Departamento de Bioquímica e Microbiologia, UNESP
}

\begin{abstract}
Resumo: Fibras de sisal com quatro tratamentos, a saber: fibra lavada com água, lavada com cicloexano/etanol, tratamento alcalino $(\mathrm{NaOH})$ e tratamento com peróxido alcalino (branqueamento), foram incorporadas na blenda amido termoplástico/policaprolactona 80/20 (TPS/PCL). As propriedades morfológicas, mecânicas e térmicas dos biocompósitos TPS/PCL/Sisal foram analisadas. Os compósitos com a fibra branqueada apresentaram os melhores resultados de resistência à tração e estabilidade térmica. Verificou-se também melhora da adesão fibra-matriz no compósito com a fibra branqueada, com aumento de $145 \%$ na resistência à tração.
\end{abstract}

Palavras-chave: Sisal, tratamento superficial, compósitos.

\section{Effect of Fiber Treatments on Properties of Thermoplastic Starch/Polycaprolactone/Sisal Biocomposites}

Abstract: Sisal fibers treated with four methods, namely washing with water, washing with cyclohexane/ethanol, alkali treatment (NaOH) and bleaching (alkaline peroxide treatment), were incorporated in thermoplastic starch/polycaprolactone 80/20 (TPS/PCL) samples. Morphological, mechanical and thermal properties of TPS/PCL/Sisal biocomposites were analysed. The best results were obtained with the composite using the bleached fiber, which had improved tensile strength and thermal stability. An increased adhesion between the fiber and matrix was also observed with the bleached fiber, with $145 \%$ increase in the tensile strength

Keywords: Sisal, superficial treatment, composites.

\section{Introdução}

O uso em compósitos poliméricos de fibras lignocelulósicas naturais como sisal, juta, bagaço de cana, curauá, etc., têm sido bastante promissor no uso em compósitos poliméricos, pois estas exibem excelentes propriedades mecânicas, redução do impacto ambiental dos compósitos e melhora da biodegradabilidade ${ }^{[1-4]}$.

A fibra de sisal (Agave sisalana) corresponde a $70 \%$ da produção comercial de todas as fibras desse tipo no mundo. Os compósitos reforçados com fibras de sisal se destacam por apresentarem alta resistência ao impacto e boas propriedades de resistência à tração e flexão ${ }^{[4,5]}$. Isto é atribuído ao fato da fibra de sisal apresentar um dos maiores valores de módulo de elasticidade e de resistência entre as fibras naturais ${ }^{[4]}$.

As propriedades dos compósitos poliméricos dependem de alguns fatores como adesão fibra-matriz, orientação da fibra, volume da fibra, etc. Para melhorar a adesão fibra-matriz, uma das alternativas é modificar química ou fisicamente as fibras antes da incorporação à matriz polimérica. Tratamentos químicos, tais como o tratamento alcalino com $\mathrm{NaOH}$, os quais removem as impurezas superficiais, induzem a uma superfície rugosa das fibras, melhorando a adesão fibra-matriz e, conseqüentemente, as propriedades mecânicas do compósito ${ }^{[7,8]}$.

O uso de fibras de sisal para obtenção de compósitos poliméricos tem sido estudado por Mattoso et al. ${ }^{[8]}$, os quais verificaram que fibras de sisal tratadas com isocianato aplicadas em polietileno de baixa densidade (LDPE) apresentaram melhora da adesão, verificando que as propriedades dinâmico mecânicas foram fortemente influenciadas pela orientação das fibras. Sreekumar et al. ${ }^{[9]}$, verificaram que os compósitos de sisal com poliéster, cuja fibra de sisal foi tratada com $\mathrm{NaOH}$, apresentaram melhor resultado de módulo elástico e resistência à tração, em relação aos compósitos com fibras tratadas com silano, permanganato, cloreto de benzila e tratamento térmico a $100{ }^{\circ} \mathrm{C}$. Esses resultados se devem à fibrilação, que aumenta a superfície de contato e ao aumento da cristalinidade da fibra, favorecendo a adesão da matriz/fibra no compósito. Iozzi et al. ${ }^{[6]}$ estudaram a influência do sisal nas propriedades dos compósitos de borracha nitrílica, observando que a fibra lavada com água apresentou maior adesão na matriz em relação à fibra não lavada. $O$ tratamento de lavagem das fibras contribuiu para a eliminação de resíduos indesejáveis da superfície das fibras, ativando a superfície da fibra, para a sua utilização em compósitos poliméricos. A incorporação de fibras de sisal sem prévio tratamento em blendas de derivados de celulose/amido foi estudada por Alvarez e Vázquez ${ }^{[10]}$. Os autores observaram que a adição da fibra de sisal não interferiu na degradação térmica da blenda.

O amido é um dos polímeros naturais mais estudados como aplicação de material plástico. O amido com plastificante, chamado de amido termoplástico (TPS) é obtido após a adição de plastificantes, como o glicerol e com a aplicação de energia termomecânica em contínuo processo de extrusão, perdendo assim, a sua estrutura original granular ${ }^{[11,12]}$.

Relacionado às blendas de amido termoplástico (TPS) com policaprolactona (PCL), um polímero sintético, alifático e biodegradável, Pérez et al. ${ }^{[21]}$ estudaram as propriedades mecânicas de blendas de TPS/PCL com a incorporação de nanoargila. Os autores verificaram que a adição da nanoargila diminuiu $300 \%$ a 
resistência à tração, conseqüentemente, melhorando o módulo de deformação.

Esse trabalho teve como objetivo avaliar o efeito na blenda TPS/ PCL 80/20 a adição de $10 \%$ em massa de fibra de sisal na blenda TPS/PCL 80/20, após diferentes tratamentos como, fibra lavada com água, fibra lavada com cicloexano/etanol, fibra após tratamento alcalino $(\mathrm{NaOH})$ e fibra após tratamento com peróxido alcalino (branqueamento), através de estudos por Microscopia Eletrônica de Varredura (MEV), Ensaio Mecânico de Tração, Calorimetria Exploratória Diferencial (DSC) e Termogravimetria (TG).

\section{Materiais e Métodos}

\section{Materiais}

As fibras de sisal foram fornecidas pela Embrapa Algodão (Campina Grande - PB, Brasil). Os reagentes empregados para os diferentes tratamentos das fibras foram: solução de cicloexano/ etanol 1:1 (v/v) Synth/Qhemis, solução de peróxido de hidrogênio $\left(\mathrm{H}_{2} \mathrm{O}_{2} 16 \%\right.$ (v/v) Nuclear) e solução de hidróxido de sódio $(\mathrm{NaOH}$ 5 wt. (\%) Qhemis).

O amido de milho utilizado foi o Amidex ${ }^{\circledR} 3001$, gentilmente cedido pela Corn Products e o PCL CAPA ${ }^{\circledR}$ 6500, da Perstorp, Mw $84500 \pm 1000$.

\section{Métodos}

\section{Tratamento das fibras de sisal}

Fibra de sisal lavada com $\mathrm{H}_{2} \mathrm{O}$

Aproximadamente $10 \mathrm{~g}$ de fibra de sisal moída e peneirada (16 mesh), com aproximadamente $3 \mathrm{~cm}$ de comprimento, foram adicionados em $200 \mathrm{~mL}$ de água destilada, a $80^{\circ} \mathrm{C}$, com agitação magnética durante 1 hora.

A fibra foi seca em estufa com circulação de ar a $50{ }^{\circ} \mathrm{C}$ até massa constante.

\section{Fibra de sisal lavada com cicloexano/etanol 1:1 (v/v)}

As fibras de sisal (10 g) foram colocadas em $200 \mathrm{~mL}$ da solução de cicloexano/etanol 1:1, durante 48 horas em soxhlet e por mais 4 horas com água destilada.

A fibra foi seca em estufa com circulação de ar a $50{ }^{\circ} \mathrm{C}$ até massa constante.

\section{Branqueamento das fibras de sisal com peróxido alcalino}

O branqueamento das fibras de sisal deu-se em duas etapas:

i) Tratamento alcalino $(\mathrm{NaOH})$

Aproximadamente $10 \mathrm{~g}$ da fibra moída e peneirada (16 mesh) foram colocados em um béquer contendo $200 \mathrm{~mL}$ de solução de hidróxido de sódio $(\mathrm{NaOH} 5 \%)$ à temperatura de $90{ }^{\circ} \mathrm{C}$. O sistema permaneceu sob constante agitação por 60 minutos. Após o resfriamento à temperatura ambiente, procedeu-se a filtração sob vácuo. O material sólido retido foi lavado com água destilada até que esta atingisse o $\mathrm{pH}$ neutro. A fibra foi seca em estufa com circulação de ar a $50{ }^{\circ} \mathrm{C}$ até massa constante.

ii) Branqueamento com solução de peróxido alcalino

Aproximadamente $5 \mathrm{~g}$ da fibra de sisal tratada com $\mathrm{NaOH}$ foram colocados em uma mistura de peróxido de hidrogênio $\left(\mathrm{H}_{2} \mathrm{O}_{2} 16 \%\right)$ e hidróxido de sódio $(\mathrm{NaOH} 5 \%)$ à temperatura de $55{ }^{\circ} \mathrm{C}$. O sistema permaneceu sob constante agitação por 90 minutos. Após o resfriamento à temperatura ambiente procedeu-se a filtração sob vácuo lavando-se o material com água destilada até que esta atingisse a neutralidade. A fibra foi seca em estufa com circulação de ar a $50{ }^{\circ} \mathrm{C}$ até massa constante.

\section{Preparo dos biocompósitos e de corpos de prova}

Os biocompósitos foram obtidos por meio de processamento em misturador de câmara interna em um reômetro Haake Rheomix 600, nas seguintes condições: rotores tipo "roller" rotação dos rotores $60 \mathrm{rpm}$, tempo de mistura de 6 minutos, temperatura de processamento de $150{ }^{\circ} \mathrm{C}$. Foi realizada uma mistura prévia dos componentes antes da alimentação no reômetro, nas seguintes proporções: amido termoplástico 72 wt. (\%), sendo glicerol 18 wt. (\%), água 10 wt. (\%), 0,2 wt. (\%) de ácido esteárico, PCL 18 wt. (\%), fibra de sisal 10 wt. (\%), resultando em uma composição final de $90 \%$ de TPS/PCL 80:20 (m/m) e 10\% de fibra de sisal.

Os biocompósitos foram posteriormente moldados por compressão a $150{ }^{\circ} \mathrm{C}$ em moldes ASTM D638-03 ${ }^{[25]}$ para obtenção de corpos de prova para os ensaios mecânicos, morfológicos e térmicos.

\section{Caracterizações dos biocompósitos}

\section{Caracterização morfológica por Microscopia Eletrônica de Varredura (MEV)}

As morfologias das superfícies das fibras de sisal e a superfície de fratura criogênica dos compósitos foram investigadas utilizando-se um microscópio Carl Zeiss DSM 940-A, à 5 kV. Os compósitos foram fraturados em nitrogênio líquido e acoplados nos porta-amostras ("stubs"). As amostras foram recobertas com uma fina camada de ouro $( \pm 15 \mathrm{~nm})$.

Termogravimetria (TG)

A temperatura de degradação termooxidativa dos compósitos foi avaliada empregando-se a técnica de termogravimetria utilizando-se um equipamento da TA Q500 (TA Instruments) nas seguintes condições: massa: 10,00 \pm 0,50 mg; atmosfera: ar sintético; fluxo $60 \mathrm{~mL} / \mathrm{min}$; razão de aquecimento: $10{ }^{\circ} \mathrm{C} / \mathrm{min}$; intervalo de temperatura: 25 a $600{ }^{\circ} \mathrm{C}$ e porta amostra de platina.

\section{Calorimetria Exploratória Diferencial (DSC)}

Os compósitos foram analisados por Calorimetria Exploratória Diferencial (DSC), em equipamento DSC Q100 (TA Instruments), nas seguintes condições: massa $6,00 \pm 1 \mathrm{mg}$, nitrogênio, fluxo $60 \mathrm{~mL} / \mathrm{min}$, em duas etapas consecutivas:

- Isoterma de 3 minutos a $-80^{\circ} \mathrm{C}$;

- Aquecimento: razão de aquecimento $10^{\circ} \mathrm{C} / \mathrm{min}$, de -80 a $150{ }^{\circ} \mathrm{C}$;

\section{Ensaio mecânico de tração}

As amostras moldadas por compressão foram avaliadas em ensaios mecânicos sob tração para a avaliação das propriedades da resistência mecânica sob tração, permitindo-se avaliar o desempenho mecânico dos materiais produzidos e comparar o efeito dos tratamentos das fibras de sisal nos compósitos estudados.

Ensaios mecânicos de tração foram realizados em um equipamento EMIC DL3000, seguindo a norma ASTM D638 ${ }^{[25]}$. Os ensaios foram realizados com velocidade de $10 \mathrm{~mm} / \mathrm{min}$ e com célula de carga de $50 \mathrm{kgf}$.

\section{Resultados e Discussões}

A morfologia da superfície longitudinal das fibras tratadas é mostrada na Figura 1. A fibra lavada com água (Figura 1a) ainda mostra resíduos de beneficiamento da fibra. Esses resíduos diminuem a adesão quando utilizadas em compósitos ${ }^{[6,13]}$. As fibras lavadas com cicloexano/etanol e $\mathrm{NaOH}$ (Figura 1b, c) apresentam as células de parênquima, não observando diferença significativa entre elas. Observa-se que essas fibras apresentam os feixes unidos pelos componentes não fibrosos (hemicelulose e lignina), formando uma compacta estrutura. A fibra tratada com $\mathrm{NaOH}$ e submetida ao tratamento com peróxido alcalino (branqueada) (Figura 1d) mostra que grande parte destes componentes foram removidos, assim como 


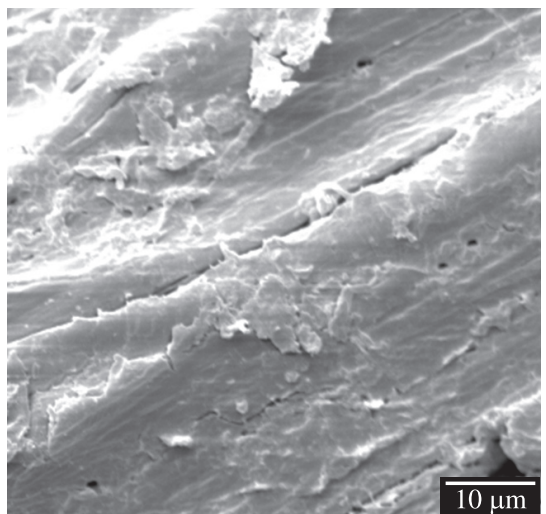

(a)

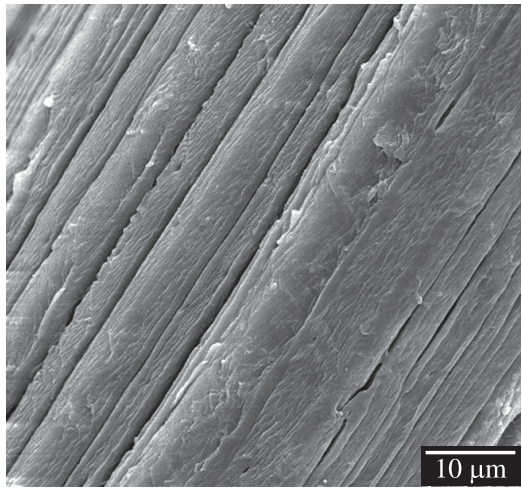

(d)

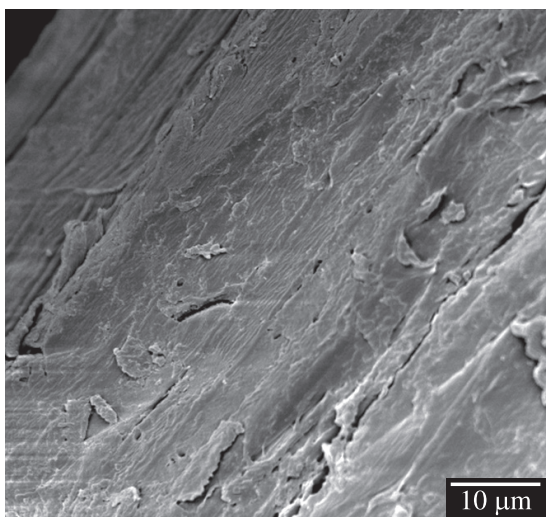

(b)

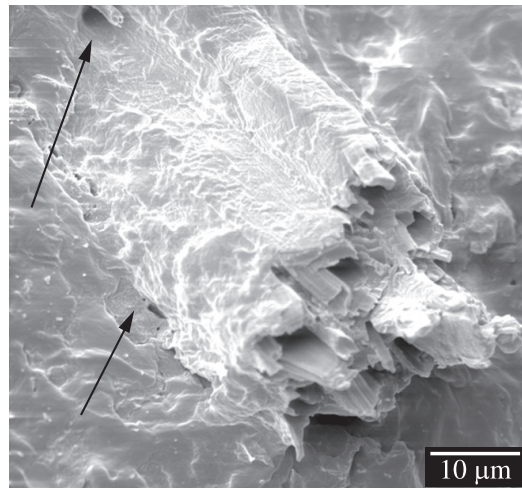

(e)

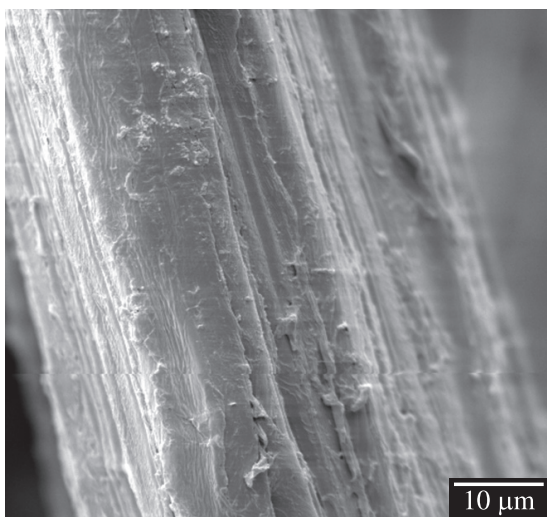

(c)

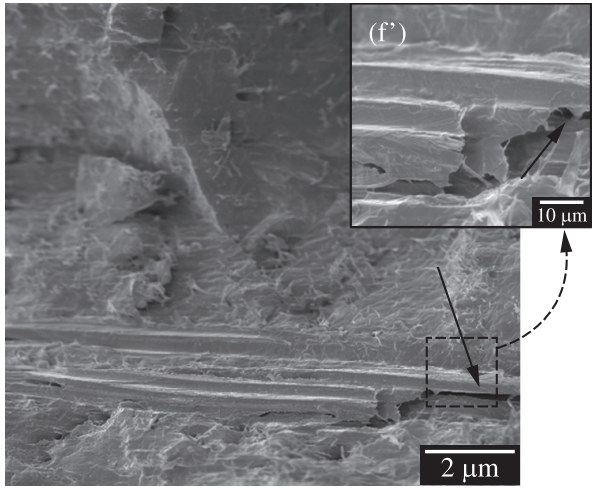

(f)

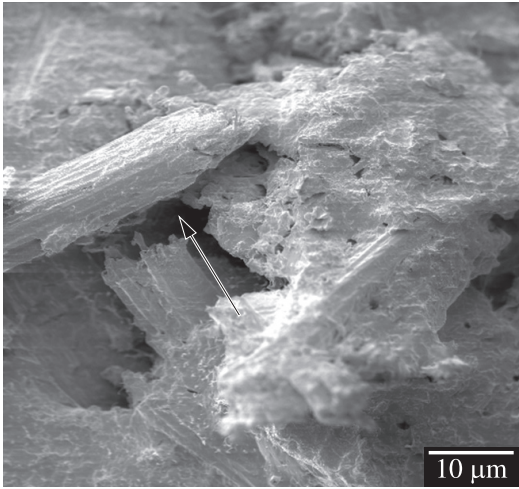

(g)

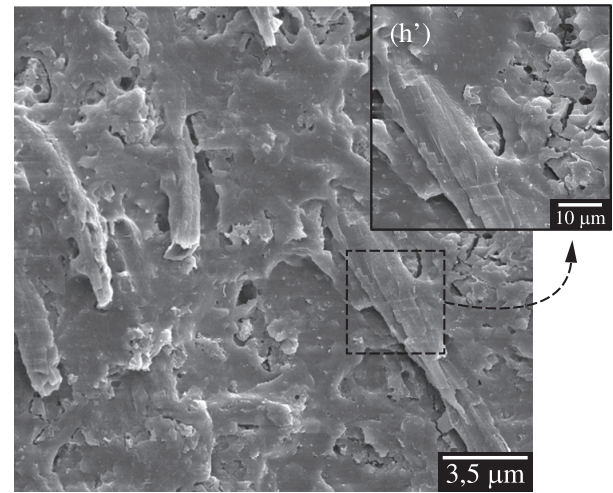

(h)

Figura 1. MEV das fibras de sisal a) lavadas com água (500 X); b) lavadas com cicloexano/etanol (1000 X); c) tratadas com NaOH (1000 X); d) após o branqueamento com peróxido alcalino (1000 X) e dos compósitos de fibras de sisal; e) lavadas com água (1000 X); f) lavadas com cicloexano/etanol (200 X); f') lavadas com cicloexano/etanol (1000 X); g) tratadas com NaOH (1000 X); h) após o branqueamento com peróxido alcalino (350 X) e h') após o branqueamento com peróxido alcalino (1000 X).

observado por Kalia et al. ${ }^{[13]}$. Os feixes de fibras tornam-se mais individualizados e microfibrilas podem ser visualizadas (Figura 1d). Na Figura 1e é apresentada a blenda TPS/PCL com a incorporação das fibras de sisal lavadas com água. Em seguida, é apresentado o compósito com as fibras de sisal lavadas com cicloexano-etanol (Figura 1f) e Figura 1f' em aumento maior, para melhor visualização e o compósito com as fibras de sisal tratada com $\mathrm{NaOH}$ (Figura 1g). Observou-se que as fibras de sisal com esses tratamentos não apresentaram boa adesão, verificando-se vazios na interface entre a fibra e a matriz, indicados por setas nas figuras. Na Figura 1h, em que se observa o compósito com o tratamento da fibra de sisal com peróxido alcalino, houve melhora da adesão da fibra na matriz, conforme indicado pelas setas.

Os valores de tensão e deformação na ruptura dos polímeros e compósitos com as fibras após diferentes tratamentos químicos são apresentados na Tabela 1. As propriedades mecânicas dos compósitos são fortemente influenciadas pela adesão entre a matriz e a fibra.

O compósito com a fibra tratada com peróxido alcalino $\left(\mathrm{NaOH}+\mathrm{H}_{2} \mathrm{O}_{2}\right)$ apresentou melhor efeito na resistência à tração que nos demais tratamentos, indicando melhor adesão e dispersão da fibra no material polimérico. 
Tabela 1. Propriedades mecânicas obtidas por ensaio de tração com diferentes tratamentos das fibras de sisal.

\begin{tabular}{lccc}
\hline \multicolumn{1}{c}{ Amostras } & Resistência à tração (MPa) & Deformação na ruptura (\%) & Módulo elástico (MPa) \\
\hline TPS & $0,8 \pm 0,05$ & $12,5 \pm 2,65$ & $154,6 \pm 11,70$ \\
PCL & $33,1 \pm 8,48$ & $798,2 \pm 119,04$ & $214,8 \pm 56,14$ \\
TPS/PCL & $2,2 \pm 0,09$ & $11,9 \pm 0,24$ & $152,0 \pm 9,69$ \\
TPS/PCL/Sisal ( $\left.\mathrm{H}_{2} \mathrm{O}\right)$ & $2,6 \pm 0,37$ & $6,5 \pm 0,44$ & $133,0 \pm 16,32$ \\
TPS/PCL/Sisal(Cicloexano/etanol) & $1,8 \pm 0,57$ & $6,0 \pm 0,57$ & $116,0 \pm 5,24$ \\
TPS/PCL/Sisal (NaOH) & $2,8 \pm 0,51$ & $4,8 \pm 0,45$ & $132,0 \pm 24,80$ \\
TPS/PCL/Sisal $\left(\mathrm{NaOH}+\mathrm{H}_{2} \mathrm{O}_{2}\right)$ & $3,7 \pm 0,27$ & $3,6 \pm 0,21$ & $137,0 \pm 23,50$ \\
\hline
\end{tabular}

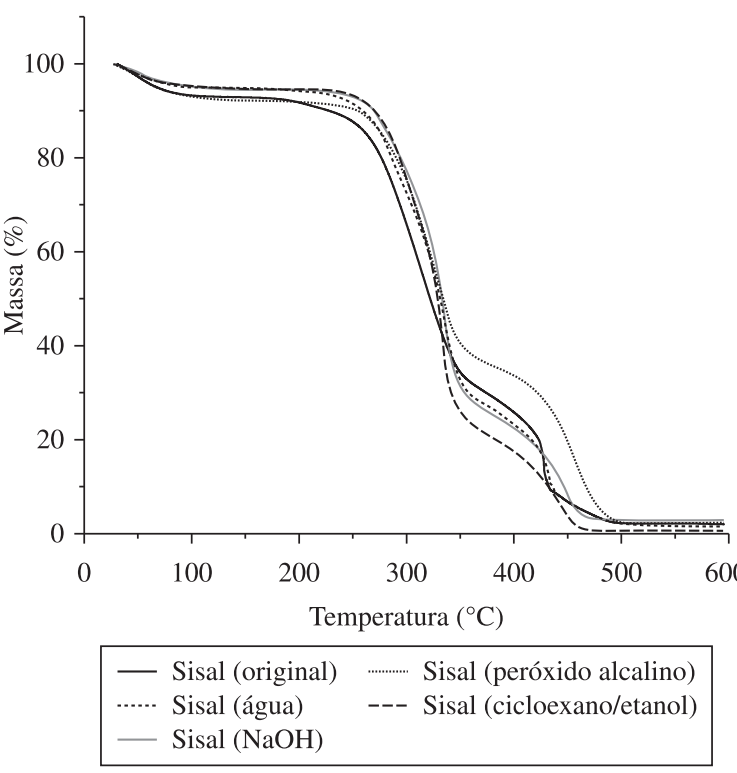

(a)

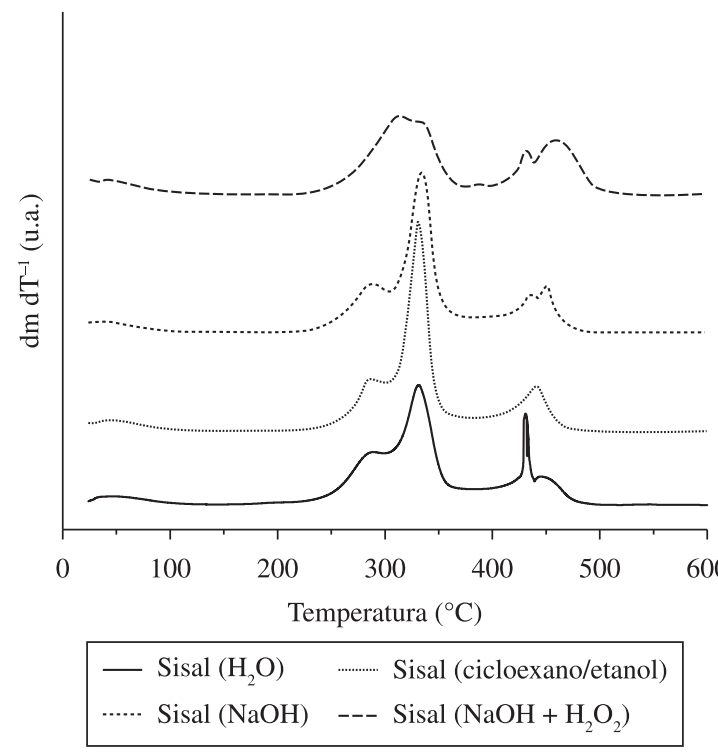

(b)

Figura 2. Curvas (a) TG e (b) DTG das fibras de sisal: lavada com água, cicloexano/etanol, tratadas com $\mathrm{NaOH}(\mathrm{NaOH})$ e branqueada $\left(\mathrm{NaOH}+\mathrm{H}_{2} \mathrm{O}_{2}\right)$.

Segundo Rosa et al. ${ }^{[14]}$, o tratamento alcalino $(\mathrm{NaOH})$ promove molhabilidade e melhor adesão fibra-matriz, permitindo eficiente transferência de tensão entre a matriz e as fibras. O tratamento alcalino remove as impurezas e promove a desfibrilação, aumentando a área superficial efetiva, conseqüentemente, propicia melhor adesão fibra-matriz e um aumento nas propriedades mecânicas ${ }^{[13,14]}$. Nesse trabalho, observou-se significativa melhora na resistência à tração com compósito com a fibra branqueada (peróxido alcalino), indicando melhora da adesão fibra-matriz.

A deformação na ruptura diminuiu com a melhora da tensão na ruptura, o que se pode ter ocorrido devido ao aumento de rigidez da fibra e ao aumento de cristalinidade, após a perda de estruturas presentes na parede celular da fibra, tal como reportado por Beckermann et al. ${ }^{[15]}$.

Quanto ao módulo elástico, não é observado diferença entre os compósitos após os diferentes tratamentos das fibras de sisal.

O estudo de termogravimetria (Figura 2) mostrou que a fibra de sisal apresenta perda de massa na faixa da temperatura ambiente até $150{ }^{\circ} \mathrm{C}$ atribuído à perda de voláteis, de 200 a $370{ }^{\circ} \mathrm{C}$, atribuídos principalmente à degradação da hemicelulose e celulose ${ }^{[16]}$ com pequena parte de degradação de lignina, e de 370 a $500{ }^{\circ} \mathrm{C}$ correspondente principalmente a lignina, pois esta apresenta degradação lenta, numa ampla faixa de temperatura, sendo o componente mais difícil de ser decomposto ${ }^{[17]}$.

A hemicelulose (vários sacarídeos) inicia a sua degradação térmica facilmente, com perda de massa entre $220-315^{\circ} \mathrm{C}^{[17]}$, tendo o seu máximo de perda de massa em $280{ }^{\circ} \mathrm{C}$.
Tabela 2. Temperatura inicial de degradação termoxidativa $\left(\mathrm{T}_{\mathrm{id}}\right)$ para as fibras de sisal e compósitos, obtidas via termogravimetria.

\begin{tabular}{cc}
\hline Amostra & $\begin{array}{c}\text { Temperatura inicial de } \\
\text { degradação térmica }\left(\mathbf{T}_{\text {id }}\right)\left({ }^{\circ} \mathbf{C}\right)\end{array}$ \\
\hline Sisal (original) & 254 \\
Sisal $\left(\mathrm{H}_{2} \mathrm{O}\right)$ & 258 \\
Sisal (cicloexano/etanol) & 303 \\
Sisal (NaOH) & 264 \\
Sisal (peróxido alcalino) & 268 \\
TPS/PCL & 282 \\
TPS/PCL/Sisal $\left(\mathrm{H}_{2} \mathrm{O}\right)$ & 252 \\
TPS/PCL/Sisal & 282 \\
(cicloexano/etanol) & 274 \\
TPS/PCL/Sisal (NaOH) & \\
TPS/PCL/Sisal & 293 \\
(peróxido alcalino)
\end{tabular}

Neste trabalho, verificou-se que as amostras de fibras tratadas apresentaram perda de massa em temperatura superior às amostras de fibras de sisal original (Tabela 2), indicando a perda de hemicelulose e parte da lignina, tornando a fibra mais rígida. Martin et al. ${ }^{[18]}$ estudaram a degradaçao térmica da fibra de sisal, após a remoção da hemicelulose, holocelulose, lignina e celulose. Verificaram que a celulose e a hemicelulose degradam em temperatura inferior à fibra de sisal original, o que pode ser atribuído a remoção da lignina. 
Na Tabela 3 é apresentado o grau de cristalinidade dos compósitos, a partir dos valores de $\Delta \mathrm{H}_{\mathrm{m}}$ obtidos das análises de DSC, segundo a Equação 1.

$$
\% \chi_{c}=\frac{\Delta H_{f}}{\Delta H_{100 \%}} \times 100
$$

onde:

$\Delta \mathrm{H}_{\mathrm{m}}=$ entalpia de fusão $\left[\mathrm{J}^{\mathrm{g}} \mathrm{g}^{-1}\right]$;

$\Delta \mathrm{H}_{\mathrm{m}}{ }^{\circ}=136 \mathrm{~J}^{\mathrm{o}} \mathrm{g}^{-1}$, que é a entalpia de fusão supondo o polímero $100 \%$ cristalino $^{[24]}$.

Observou-se que o compósito com a fibra branqueada apresentou uma discreta diminuição na entalpia de fusão e menor grau de cristalinidade, o que sugere que a adição da fibra tratada permite que a cristalização ocorra a uma temperatura mais baixa, apresentando espessuras lamelares menores, devido à interação fibra-matriz (Figura 4 e Tabela 3).

\section{Conclusões}

Foi importante verificar a influência de diferentes tratamentos aplicados às fibras de sisal antes de sua incorporação na blenda de TPS/PCL, no sentido de melhorar as interações interfaciais entre a fibra e a matriz e, portanto, de suas propriedades mecânicas. Os compósitos com as fibras branqueadas apresentaram melhora da resistência à tração que os demais compósitos, melhora da estabilidade térmica e, portanto, melhor adesão fibra-matriz. Esses resultados sugerem que a fibra de sisal branqueada pode ser aplicada na blenda TPS/PCL como agente de reforço.

\section{Agradecimentos}

Os autores agradecem à Fapesp (Processo nº 2008/08264-9), MCT/FINEP, CNPq (483265/2007-1) e à EMBRAPA.

\section{Referências Bibliográficas}

1. Islam, M. S.; Pickering, K. L. \& Foreman, N. J. - Polym. Degrad. Stabil., 95, p.59 (2010).

2. Xu, Y.; Wu, Q.; Lei, Y. \& Yao, F. - Bioresour. Technol., doi:10.1016/j. biortech.2009.12.072. (2010).

3. Corradini, E.; Ito, E. N.; Marconcini, J. M.; Rios, C. T.; Agnelli, J. A. M. \& Mattoso, L. H. C. Polymer Testing, 28, p.183 (2009).

4. Satyanarayana, K. G.; Arizaga, G. G. C. \& Wypych, F. - Biodegr. Progr. Polym. Sci., 34, p.982 (2009).

5. Martin, A. R.; Martins, M. A.; Mattoso, L. H. C. \& Silva, O. R. R. F. Polímeros, 19, p.40 (2009).

6. Iozzi, M. A.; Martins, M. A. \& Mattoso, L. H. C. - Polímeros, 14, p.93 (2004).
7. Corradini, E.; Morais, L. C.; Rosa, M. F.; Mazzetto, S. E.; Mattoso, L. H. C. \& Agnelli, J. A. M. A. - Macromol. Symp., 245-246, p.558 (2006).

8. Mattoso, L. H. C.; Ferreira, F. C. \& Curvelo, A. A. S. - "Sisal Fiber: Morphology and Applications in Polymer Composites", in: "Lignocellulosic-Plastic Composites", A. L. Leão; F. X. Carvalho \& E. Frollini (Eds.), USP, São Paulo, p.241 (1997).

9. Sreekumar, P. A.; Thomas, S. P.; Saiter, J. M.; Joseph, K.; Unnikrishnan, G. \& Thomas, S. - Composites: Part A, 40, p.1777 (2009).

10. Alvarez, V. A. \& Vázquez, A. - Polym. Degrad. Stabil., 84, p.13 (2004).

11. Averous, L.; Boquillon, N. Carbohyd. Polym., 56, p.111 (2004).

12. Corradini, E.; Carvalho, A. J. F.; Curvelo, A. A. S.; Agnelli, J. A. M. \& Mattoso, L. H. C. - Mat. Res., 10, p.227 (2007).

13. Kalia, S.; Kaith, B. S. \& Kaur, I. - Polym. Eng. Sci., 49, p.1253 (2009).

14. Rosa, M. F.; Chiou, B. S.; Medeiros, E. S.; Wood, D. F.; Williams, T. G.; Mattoso, L. H. C.; Orts, W. J. \& Imam, S. H. - Bior. Technol., 100, p.5196 (2009).

15. Beckermann, G. W. \& Pickering, K. L. - Composites: Part A, 39, p.979 (2008).

16. Di Franco, C. R.; Cyras, V. P.; Busalmen, J. P.; Ruseckaite, R. A. \& Vásquez, A. - Polym. Degrad. Stabil., 86, p.95 (2004).

17. Yang, H.; Yan, R.; Chen, H.; Lee, D. H. \& Zheng, C. - Fuel, 86, p.1781 (2007).

18. Martin, A. R.; Martins, M. A.; Da Silva, O. R. R. F. \& Mattoso, L. H. C. - Thermoch. Acta., 506, p.14 (2010).

19. Wei, C.;Zeng, M.;Xiong, X.M.\&Zhang,F.A.-Plastic. RubberCompos. 39, p.61 (2010).

20. Guinesi, L. S.; Róz, A. L.; Corradini, E.; Mattoso, L. H. C.; Teixeira, E. M. \& Curvelo, A. S. - Thermoch. Acta, 447, p.190 (2006).

21. Pérez, C. J.; Alvarez, V. A. \& Vázquez, A. - Mater. Sci. Eng. A, 480, p.259 (2008)

22. Vertuccio, L.; Gorrasi, G.; Sorrentini, A. \& Vittoria, V. - Carbohyd. Polym., 75, p.172 (2009).

23. Liu, H.; Xie, F.; Yu, L.; Chen, L. \& Li, L. - Progress. Polym. Sci., 34, p.1348 (2009).

24. Kesel, C.; Vander Wauven, C. \& David, C. - Polym. Degrad. Stabil., 55, p.107 (1999).

25. ASTM. - "ASTM D 638-03 - Standard Test Method for Tensile Properties of Plastics" (2003).

Enviado: 10/06/10 Reenviado: $12 / 10 / 10$

Aceito: 06/12/10

DOI: $10.1590 / \mathrm{S} 0104-14282011005000039$ 